\title{
Preoperative Cardiac Stress Tests Ordered in the Preoperative Evaluation Clinic: A Retrospective Review of Ordering Patterns
}

\author{
Adam S. Weinstein ${ }^{\mathrm{a}, \mathrm{b}}$, Angela M. Bader ${ }^{\mathrm{a}}$, Richard D. Urman ${ }^{\mathrm{a}}$, \\ David L. Hepner ${ }^{\text {a }}$ John A. Fox ${ }^{\text {a }}$
}

\begin{abstract}
Background: The role of anesthesiologists has expanded from operating rooms to preoperative evaluation clinics. This role involves performing preoperative cardiovascular evaluation and optimization of patients before elective surgery, which can include ordering cardiac stress tests. We aimed to study the ordering patterns by anesthesiologists for preoperative cardiac stress tests, focusing on whether societal and institutional guidelines and recommendations were used. Choice of type of cardiac stress test was also examined.
\end{abstract}

Methods: A single center retrospective chart review from December 1, 2005 to May 31, 2015 was performed on 492 patients who had a cardiac stress test ordered by an anesthesiologist. Patients were categorized by indication for ordering the cardiac stress test based on societal practice guidelines, institutional guidelines or other relevant reasons at the time of patient encounter. Those "other" category cardiac stress tests were assessed for indication and evaluated by physician peer review to see if there was peer agreement for being appropriately ordered. Exercise electrocardiography (ECG) cardiac stress tests ordered were evaluated for appropriateness based on baseline resting ECG findings. Patients with left bundle branch block (LBBB) or right ventricular (RV) pacing were evaluated for appropriateness of proper cardiac stress test modality based on whether a pharmacological vasodilator cardiac stress test was ordered.

Results: Analysis of the cardiac stress tests ordered showed that $43 \%$ were ordered according to American College of Cardiology/American Heart Association guidelines, 29\% were ordered according to institutional guidelines, and $28 \%$ were categorized as "other". Of the $28 \%$ "other" cardiac stress tests, $53 \%$ were in agreement for ordering by peer review. Sixty-four exercise ECG cardiac stress tests were

Manuscript submitted December 17, 2018, accepted January 18, 2019

aDepartment of Anesthesiology, Perioperative and Pain Medicine, Brigham and Women's Hospital/Harvard Medical School, 75 Francis Street, CWN-L1, Boston, MA 02115, USA

${ }^{\mathrm{b} C}$ Corresponding Author: Adam S. Weinstein, Department of Anesthesiology, Perioperative and Pain Medicine, Brigham and Women's Hospital, 75 Francis Street, CWN-L1, Boston, MA 02115, USA.

Email: asweinstein@bwh.harvard.edu

doi: https://doi.org/10.14740/cr821 ordered, of which 58\% were appropriate based on having no baseline resting ECG abnormalities. Fifty-one patients were identified as having a resting ECG of LBBB or RV pacing of which $41 \%$ had an appropriate pharmacological vasodilator cardiac stress tests ordered.

Conclusions: Anesthesiologists order most preoperative cardiac stress tests according to professional societal or institutional guidelines $(72 \%)$, yet they are not always choosing the best modality of cardiac stress test. A significant portion of cardiac stress tests are ordered (28\%) based on clinical judgment, likely due to the lack of guidelines and recommendations being all-encompassing on many commonly encountered preoperative patient situations.

Keywords: Anesthesia; Preoperative; Evaluation; Cardiac testing; Stress test

\section{Introduction}

The role of the anesthesiologist has evolved from an operating room physician to a perioperative physician and this begins with evaluation of the patient in a preoperative evaluation clinic (PEC). Such visits have been associated with a decrease in postoperative hospital mortality [1].

A main focus of a PEC visit is risk stratification for cardiovascular complications and assessment if patient optimization is needed. This can lead to further cardiac testing such as cardiac stress tests. Appropriate selection of individuals for cardiac stress testing is essential to maximize positive and negative predictive value of the test. This can improve the chances of identifying patients who can undergo either medical or surgical optimization prior to an elective surgery with the intent of decreasing perioperative morbidity and mortality. The American College of Cardiology (ACC)/American Heart Association (AHA) Guidelines on Perioperative Cardiovascular Evaluation and Management of Patients Undergoing Noncardiac Surgery provide recommendations for preoperative cardiac stress tests [2]. Unfortunately, these ACC/AHA Guidelines do not encompass all of the real-life preoperative patient situations and scenarios which leave the ordering of cardiac stress tests up to the discretion of the perioperative physician.

No study to date has examined practice behaviors of anes- 
thesiologists ordering preoperative cardiac stress tests. Therefore, we performed a single-center retrospective study over a 10 -year period to examine the indications for ordering preoperative cardiac stress tests by anesthesiologists. When the indications for ordering were not according to societal guidelines or institutional guidelines, they were assessed by peer review whereby if at least two out of three of the peer-reviewing senior anesthesiologists agreed on the ordering of the stress test, it was deemed appropriate. Since there are many types of cardiac stress tests, we also examined the choice of type of cardiac stress test ordered by anesthesiologists. Specifically, we examined the ordering of two common specific types of cardiac stress tests, pharmacological vasodilator cardiac stress test and exercise electrocardiogram (ECG) stress test. We identified if the choice of those cardiac stress types were appropriate, as those tests have patient-specific factors that can invalidate them. We hypothesized that there is a significant amount of variability among anesthesiologists regarding the decision to order a test and the type of test.

\section{Materials and Methods}

The Brigham and Women's Hospital (BWH) Institutional Review Board (IRB) granted approval for the chart review, publication and waiver of individual consent. BWH uses the computer software program Precipio ${ }^{\circledR}$ (Precipio Inc, New Haven, CT) to order cardiac stress tests. Using this software, we identified patients who underwent a cardiac stress test ordered by an anesthesiologist at the BWH PEC from December 1, 2005 to May 31, $2015(\mathrm{~N}=492)$. The data extracted from the charts were de-identified and stored in an encrypted and passwordprotected Excel spreadsheet that was created specifically for this study.

Documentation regarding ordering of the cardiac stress tests was studied to identify the indication for order by the anesthesiologist. Patients undergoing cardiac stress tests without an available PEC visit note were excluded $(\mathrm{N}=96)$. In addition, patients undergoing cardiac stress tests were excluded if the PEC visit note documented a recommendation by another service, such as a cardiologist or primary care physician (PCP) to obtain the cardiac stress test $(\mathrm{N}=41)$. This was done as it was not possible to determine if the anesthesiologist would have ordered the test without the recommendation. The indications for the cardiac stress tests $(\mathrm{N}=355$, after exclusion) were classified into three categories as described in detail below: 1) Societal guidelines; 2) Institutional guidelines; or 3) Other.

\section{Societal guidelines}

The cardiac stress test indication for order was classified as "Societal guidelines" if the test was ordered according to the ACC/AHA Guidelines on Perioperative Cardiovascular Evaluation and Management of Patients Undergoing Noncardiac Surgery. This category was further subdivided into two subcategories: "Algorithm" and "ECG".

If the test was ordered according to the ACC/AHA Guide- lines on Perioperative Cardiovascular Evaluation and Management of Patients Undergoing Noncardiac Surgery Stepwise Approach to Perioperative Cardiac Assessment: Treatment Algorithm, it was classified as "Societal guidelines: Algorithm" [2]. Of note the ACC/AHA Guidelines have evolved over time with current being the 2014 guidelines (previously the 2007 ACC/AHA Guidelines [3] and 2002 ACC/AHA Guidelines [4] iterations). This evolution spans the time span of this study and the use of the time-appropriate guideline was checked.

If the test was ordered according to the ACC/AHA Guidelines on Perioperative Cardiovascular Evaluation and Management of Patients Undergoing Noncardiac Surgery: The 12-Lead Electrocardiogram Recommendations [2] which subsequently resulted in ordering an ECG that indicated a need for a cardiac stress test, it was classified as "Societal guidelines: ECG" (BWH uses both the IIa and IIb recommendations). Per BWH protocol, significant ECG findings deemed to indicate a cardiac stress are new findings of left bundle branch block (LBBB), T wave inversions in two or more consecutive leads, $\mathrm{Q}$ waves in two more consecutive leads, ST depression in two or more consecutive leads, or ST elevations in two or more consecutive leads. These ECG findings are based on wellknown markers of ischemia [5, 6]. Similarly as before, the ACC/AHA Guidelines have evolved over time with current being the 2014 guidelines (previously the 2007 ACC/AHA Guidelines [3] and 2002 ACC/AHA Guidelines [4] iterations). This evolution spans the length of this study and the use of the time appropriate guideline was checked.

\section{Institutional guidelines}

The cardiac stress test indication for order was classified as "Institutional guidelines" if an ECG was ordered if the BWH guidelines indicated a need for a cardiac stress. BWH has supplemental guidelines in addition to the ACC/AHA guidelines. Specific to this study, BWH obtains an ECG on every patient older than the age 50 years (until June 2015). This institutional specific BWH guideline is not in the ACC/AHA guidelines and was a group practice decision made to help prevent same day surgery cancelations or delays for new ECG findings discovered on the day of surgery. Table 1 shows a full list of the supplemental BWH institutional specific guidelines on ordering preoperative ECGs. Similarly, as before, ECG findings at BWH deemed to indicate a cardiac stress test are new LBBB, T wave inversions in two or more consecutive leads, Q waves in two more consecutive leads, ST depression in two or more consecutive leads, or ST elevations in two or more consecutive leads.

\section{Other}

The cardiac stress test indication for order was classified as "Other" if the cardiac stress test was ordered at the discretion of the perioperative anesthesiologist and was not according to societal guidelines or institutional guidelines. The indication for ordering the cardiac stress test by the anesthesiologist was also noted. For every "Other" classified cardiac stress test, 
Table 1. Brigham and Women's Hospital Preoperative Electrocardiogram Ordering Guidelines

Any patient over the age of 65 (updated in June 2015, previously age 50)

Any patient with a known arrhythmia

Any patient with a suspected arrhythmia by history regardless of age

Any patient with an AICD or pacemaker regardless of age

Any patient with known significant cardiac history regardless of RCRI or age

Shown is the list of institutional specific guidelines at Brigham and Women's Hospital for ordering preoperative electrocardiograms. These supplement the American College of Cardiology/American Heart Association Guidelines on Perioperative Cardiovascular Evaluation and Management of Patients Undergoing Noncardiac Surgery. AICD: automatic implantable cardioverter-defibrillator; RCRI: revised cardiac risk index.

three senior perioperative anesthesiologists (AMB, DLH, RPC) knowledgeable in the ACC/AHA and the institutional specific guidelines each reviewed the documented indication for ordering the cardiac stress test and commented either "yes" or "no" if in their own clinical judgment, they would have ordered a cardiac stress for the patient. If at least two out of three of the peer-reviewing anesthesiologists commented "yes", then the cardiac stress test was deemed "agreed upon" or "appropriate".

For a secondary analysis, we examined all cases without excluding charts that were not considered for the primary analysis. Thus, for this analysis we included all charts without exclusion, and we documented the type of cardiac stress test for all of the cardiac stress tests ordered in the study period $(\mathrm{N}=$ 492). This means no charts/cardiac stress tests were excluded, as this was an analysis of whether a proper choice type of cardiac stress test was done based on the patient's preoperative ECG. This was possible since at the PEC, a preoperative ECG was obtained prior to getting a preoperative cardiac stress test if an ECG had not already been done previously. All 492 charts were assessed if the patient's preoperative ECG had a LBBB or right ventricle (RV) pacing and then subsequently determined if the type of cardiac stress test ordered was a pharmacologic vasodilator cardiac stress test. For a different analysis, all 492 charts were assessed if the type of cardiac stress test ordered was an exercise ECG cardiac stress test. Subsequently it was determined if the preoperative resting ECG had a LBBB, T wave inversions, $Q$ waves, ST depressions or elevations. As previously stated, these ECG findings are common markers of ischemia that the BWH PEC considers invalidating for an exercise ECG cardiac stress test if found on the resting baseline ECG.

\section{Results}

A total of 492 cardiac stress tests ordered by anesthesiologists at the BWH PEC from December 1, 2005 to May 31, 2015 were identified. For 96 of the cardiac stress tests ordered the accompanying PEC visit notes were unobtainable and were not analyzed for indication. At the study's institution the transition from paper-based notes to electronic notes resulted in a loss of original paper-based notes and as a consequence some PEC visit notes were not obtainable either due to them not ever being scanned in or misclassified as a different note type in the electronic medical record. Cardiac stress tests were ordered by recommendation by another medical service for 41 patients ( 26 by cardiology, 13 by primary care physician, one by pri- mary surgical team, one by pulmonary medicine). This left 355 cardiac stress tests for analysis according to the indication for ordering (Fig. 1).

Analyzing the 355 cardiac stress tests for indication of order and categorizing them according to the previously mentioned categories, it was found that 153 (43\%) were classified as "Societal guidelines", 104 (29\%) were classified as "Institutional guidelines", and 98 (28\%) were categorized as "Other". Further breakdown of the "Societal guidelines" showed that 101 of the $153(66 \%)$ were categorized as "Societal guidelines: ECG" and 52 of the 153 (34\%) were categorized as "Societal guidelines: Algorithm" (Table 2).

For the "Other" categorized cardiac stress tests, the breakdown for indication for order is noted in Table 3. The 98 cardiac stress tests categorized as "Other" were reviewed by three senior perioperative anesthesiologists (AMB, DLH, RPC) and $52(53 \%)$ of the 98 were agreed upon as appropriate, meaning at least two of the three peer reviewers agreed they would order a cardiac stress test for that patient (Table 3). Of the "Other" cardiac stress tests, $50 \%(\mathrm{~N}=49)$ of them were unanimously agreed upon as either appropriate or inappropriate (meaning

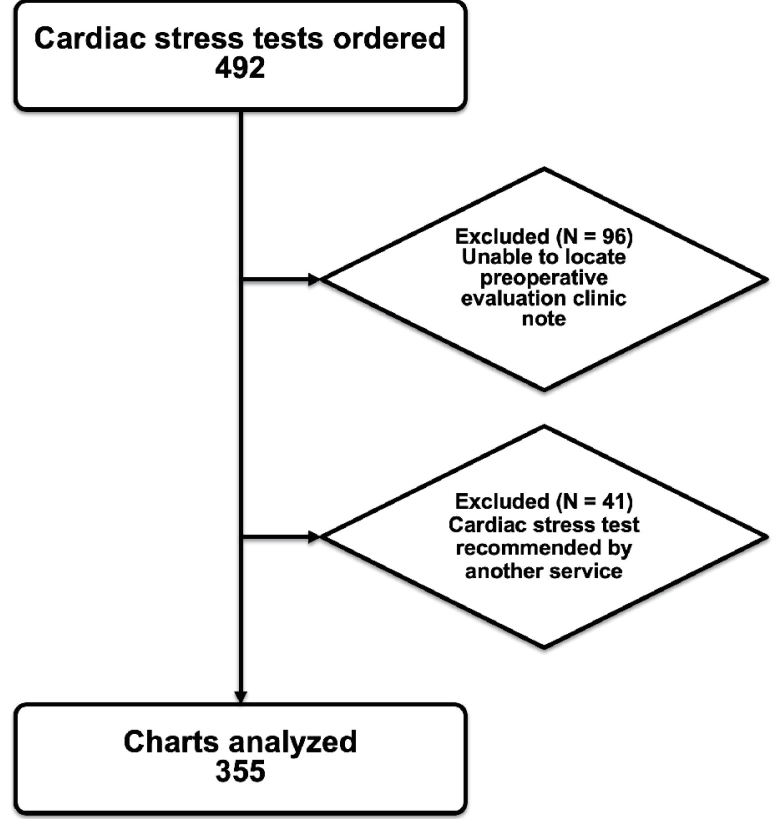

Figure 1. Cardiac stress tests ordered exclusion flow diagram (flow diagram of the charts analyzed and excluded). 
Table 2. Cardiac Stress Test Ordered by Indication

\begin{tabular}{lll}
\hline Cardiac stress ordered by indication & Number & Percent of total \\
\hline Societal guidelines & 153 & $43 \%(153 / 355)$ \\
Societal guidelines: ECG & 101 & $28 \%(101 / 355)$ \\
Societal guidelines: algorithm & 52 & $15 \%(52 / 355)$ \\
Institutional guidelines & 104 & $29 \%(104 / 355)$ \\
Other & 98 & $28 \%(98 / 355)$ \\
Charts analyzed & 355 & $100 \%(355 / 355)$ \\
\hline
\end{tabular}

Breakdown of indications for order of cardiac stress tests ordered. "Percent of total" column represents the percent of cardiac stress tests in that category (row) of all cardiac stress tests ordered. ECG: electrocardiogram.

all three peer reviewing anesthesiologists said either "yes" or "no" to being appropriate).

For analysis of the ordering of types of cardiac stress tests, specifically pharmacological vasodilator cardiac stress tests, 51 patients were identified having a resting ECG of LBBB or $\mathrm{RV}$ pacing. Of those 51 patients, the cardiac stress test type ordered was a pharmacologic vasodilator stress test in $41 \%$ (N $=21$ ) of the cases (Table 4). For analysis of the ordering of exercise ECG cardiac stress tests, 64 exercise ECG cardiac stress tests were identified. Of these cardiac stress tests, $58 \%(\mathrm{~N}=$ 37 ) of the patients had no resting ECG abnormalities to invalidate the test (Table 4 ). Alternatively phrased, $42 \%$ of exercise ECG cardiac stress tests had baseline ECG abnormalities that invalidated the test. Baseline ECG abnormalities at BWH deemed to invalidate the test are LBBB, T wave inversions in two or more consecutive leads, Q waves in two more consecutive leads, ST depression in two or more consecutive leads, or ST elevations in two or more consecutive leads.

\section{Discussion}

We performed a single center retrospective study of 492 charts over a 10 -year period analyzing the indications for ordering preoperative cardiac stress tests and choice of proper selection of cardiac stress test modality. We found that $72 \%$ of preop-

Table 3. Breakdown of Cardiac Stress Test Categorized as "Other"

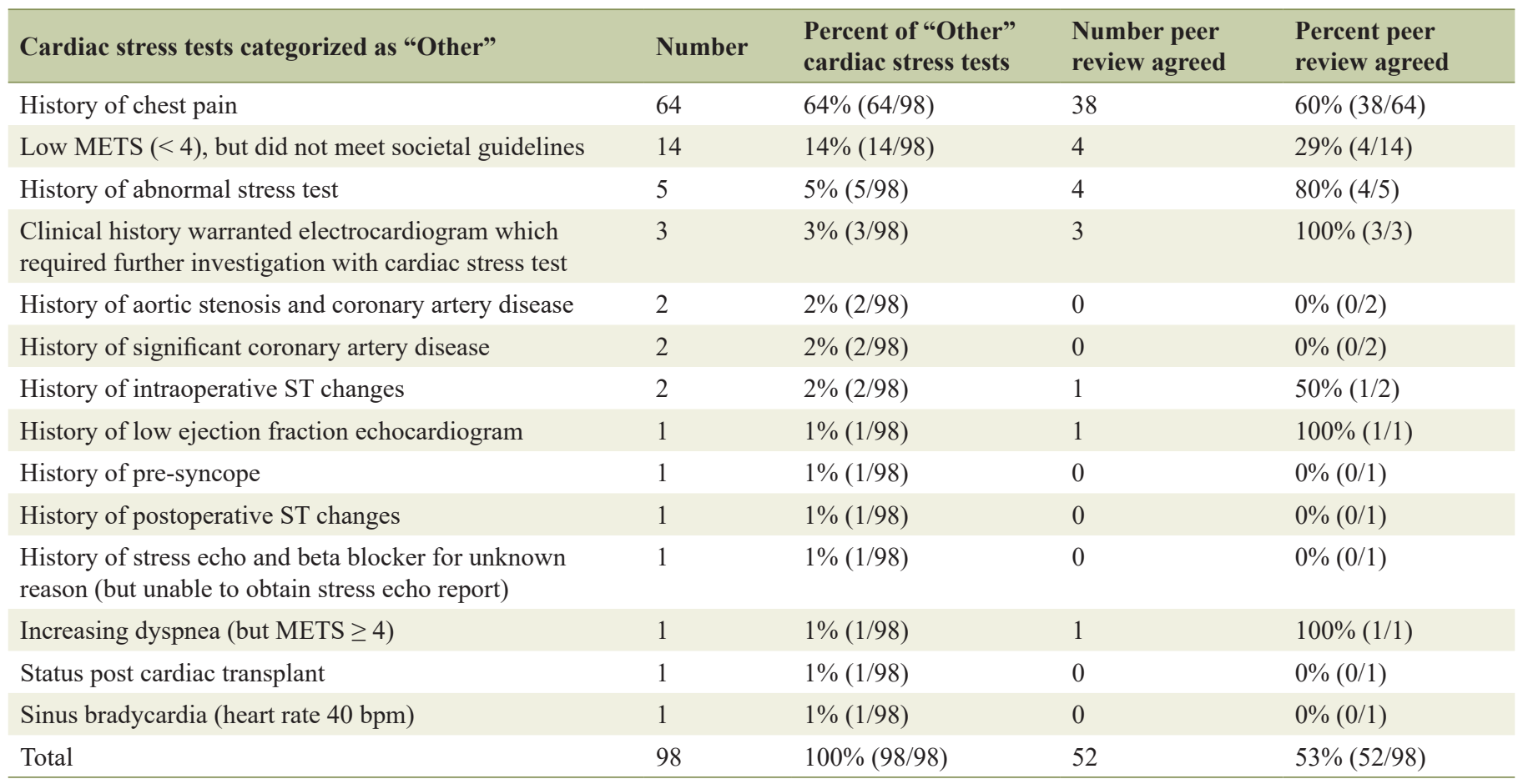

Breakdown of cardiac stress tests categorized as "Other". "Percent of "Other" cardiac stress Tests" column represents the percent of cardiac stress tests in that category (row) of cardiac stress tests categorized as "Other". "Number peer review agreed" column represents the number of cardiac stress tests of that type of indication for order (row) that by peer review was agreed upon as appropriate. "Percent peer review agreed" column represents the percent of cardiac stress tests of that type of indication for order (row) that by peer review was agreed upon as appropriate. METS: metabolic equivalents; echo: echocardiogram; bpm: beats per minute. 
Table 4. Assessment of Ordering Different Types of Cardiac Stress Tests

\begin{tabular}{|c|c|c|}
\hline & Number & Percentage of proper ordering \\
\hline Patients with LBBB or RV pacing with any type of stress test ordered & 51 & $41 \%(21 / 51)$ \\
\hline Patients with LBBB or RV pacing with pharmacological vasodilator stress test ordered & 21 & \\
\hline Exercise ECG stress tests ordered with normal resting baseline ECG & 37 & \\
\hline
\end{tabular}

Shown is the analysis of the ordering of different types of cardiac stress tests, specifically pharmacological vasodilator cardiac stress tests and exercise ECG stress tests. LBBB: left bundle branch block; RV: right ventricle; ECG: electrocardiogram.

erative cardiac stress tests ordered by anesthesiologists were consistent with societal or institutional guidelines. The largest number $(43 \%)$ of preoperative cardiac stress tests was categorized as "Societal guidelines", meaning they were ordered according to the ACC/AHA guidelines. The sub category of cardiac stress tests, "Societal guidelines: ECG" accounted for $28 \%$ of all cardiac stress tests ordered. While the ACC/AHA guidelines state that routine preoperative ECG testing is not useful in asymptomatic patients undergoing low-risk surgeries [2], anesthesiologists at our institution ordered ECGs on every patient over the age of 50 years until May 31, 2015. The decision to order these ECGs was based on the group's collective practice preferences to avoid same day cancellation of cases for previously unknown ECG abnormalities. Since June of 2015 , based on a recent study at our institution, this was changed to considering ordering ECGs on every patient over the age of 65 years, which has been shown to be an independent risk factor for significant preoperative ECGs abnormalities [7]. The "Institutional guidelines" that resulted in cardiac stress tests ordered accounted for $29 \%$ of all preoperative cardiac stress tests ordered. Summing the "Societal guidelines: ECG" and "Institutional guidelines" adds up to $57 \%$ of all cardiac stress tests ordered. This means that $57 \%$ of all cardiac stress tests were ordered as the result of preoperative ECGs obtained from societal or institutional recommendations which subsequently had markers of ischemia that triggered the ordering of a cardiac stress test. Again, those markers of ischemia were previously described in the Methods section as new findings of LBBB, T wave inversions in two or more consecutive leads, Q waves in two more consecutive leads, ST depression in two or more consecutive leads, or ST elevations in two or more consecutive leads. While there is no explicit recommendation in either the ACC/AHA guidelines or this institution's guidelines to get a cardiac stress test when a resting ECG has markers of ischemia on it, this institution's perioperative anesthesiologists at the PEC will pursue that testing. This group practice decision is a conservative practice model for several reasons. First, if those ischemic markers are seen on telemetry preoperatively without being worked up it would likely result in a case cancellation or delay on the day of surgery. Second, if the alternative path of referring to a cardiologist is taken, there is a high chance the same workup of a cardiac stress test would be pursued, as this is the typical next step in an ischemic workup. This adds to further case delay and an unnecessary cardiology consult that can be avoided if the cardiac stress test is negative. This practice model does raise the question of what is the incidence of a positive cardiac stress test that re- sults in intervention, and ultimately the utility of these tests to decrease the actual morbidity and mortality in a cost-effective manner. Unfortunately, this question is outside the scope and ability of this study and would require a large long-term multicenter study in order to identify enough positive cardiac stress tests that get subsequent assessments and care or intervention.

The remaining $28 \%$ of preoperative cardiac stress tests that were ordered, categorized as "Other", were those for indications outside of societal and institutional guidelines. This does not mean those guidelines are flawed but more likely that they are not all encompassing. Not every patient can be fitted into an algorithm and there is often insufficient evidence for ordering cardiac stress tests for many commonly encountered situations which then must rely on physician clinical judgment. Of these "Other" cardiac stress tests, 53\% were deemed appropriate by peer review. The majority $(65 \%)$ of the indications for these cardiac stress tests were for preoperative chest pain. Another large portion of these "Other" cardiac stress tests were for low functional status despite not meeting criteria by societal or institutional guidelines. Perhaps this is the patient population that fails the anesthesiologist's clinical "eye ball test", meaning they look unwell overall and have multiple comorbidities and risk factors that don't necessarily fit into the Revised (Lee) Cardiac Risk index (RCRI) that the ACC/AHA guidelines use in their Stepwise Approach to Perioperative Cardiac Assessment [8].

The question of what to do with ambulatory preoperative chest pain is a challenging one. Unlike patients presenting to a cardiologist or PCP with chest pain as the chief complaint, the perioperative physician inquires to the patient about the chest pain during the review of systems evaluation. This is a very different chest pain patient population and likely the positive predictive value of chest pain for myocardial ischemia is lower in this population than patients seeking care in other settings. Commonly, these patients have not seen a PCP in the recent past, or ever. When the preoperative clinician inquires to the patient about a history or current chest pain, it is often the first time the patient has been asked about it. Patients are commonly anxious about their upcoming surgery, which can often be perceived as palpations or chest pain. Sometimes an anxious patient will overstate symptoms so that "nothing gets missed". This makes it difficult for the perioperative anesthesiologist to decide how to work up and manage patients with ambulatory atypical or non-classical anginal chest pain symptoms. Rarely do patients tell a story of classic angina pectoris symptoms and meet all three AHA criteria for typical angina pectoris: substernal chest discomfort with a characteristic quality and duration, 
which is provoked by exertion or emotional stress, and relieved by rest or nitroglycerin [9]. There are no specific guidelines on the management of non-specific chest pain in the outpatient setting, especially those presenting for preoperative surgical evaluation. Wilhelmsen et al [10] in a long-term study analyzed men who had not consulted any physician for chest pain. The men were subsequently questioned about chest pain and evaluated and categorized by physicians into three categories: no chest pain, nonspecific chest pain or typical angina pectoris. The men classified as having nonspecific chest pain had nearly as high a cardiovascular morbidity and mortality and even higher noncardiovascular mortality compared to men who were classified as having typical angina pectoris. Of course, the perioperative physician can assess for concomitant risk factors for coronary artery disease and combine this information with a history of nonspecific chest pain to decide on further workup [11]. However, this only addresses the issue of coronary artery disease whereas the goal with the RCRI and ACC/AHA guidelines is to assess for major adverse cardiac events not just myocardial infarction from coronary artery disease [8]. Ultimately, the decision to pursue further workup may depend on the physician's clinical judgment and the overall clinical picture.

While $53 \%$ of the "Other" or non-guideline driven cardiac stress tests ordered were deemed appropriate by our peer review might seem low, the previous paragraph brings attention to the problems of preoperative cardiovascular assessment at preoperative clinics. Physician's own clinical judgment often comes into play, which is very variable. This can be seen by the fact that of all the charts reviewed by the three peerreviewing senior perioperative anesthesiologists, there was a complete agreement only $50 \%$ of the time as to whether a cardiac stress test was appropriate or not. This means that one of the three senior anesthesiologists disagreed with the other two senior anesthesiologists in $50 \%$ of the charts reviewed. The authors chose a peer review model of all anesthesiologists to get a sense if there is consensus within the specialty. Added value would come from a separate peer review by all cardiologists to see if there is consensus for ordering these "Other" cardiac stress tests. Comparing the peer reviews of the anesthesiologists to those of cardiologists would be of high interest. Both of these are protentional ideas for future studies.

Only $53 \%$ of the "Other" cardiac stress tests were deemed appropriate and only $50 \%$ of the "Other" cardiac stress tests had unanimous peer review vote which can be alarming. However, this must be put into context. During this 10 -year study the BWH PEC saw approximately 25,000 patients a year, equating to approximately 250,000 patients. After exclusions, 98 cardiac stress tests were categorized as "Other" cardiac stress tests. This approximates to less than $0.04 \%$ of the patients seen at the BWH PEC. While this approximation is more of a "guesstimate", it's important to create this context to show that the vast majority of patients and their cardiovascular assessment and workups done by the anesthesiologist are following societal and institutional direction. Based on this larger view, it can be seen that anesthesiologists are following guidelinedriven workup very well. In approximately less than $0.04 \%$ of the patient assessments is there non-guideline-driven workup. This is not to say that number of "Other" cardiac stress deemed appropriate cannot be improved. Anesthesiology subspecialty training in perioperative medicine is becoming more common and can provide additional training for assessment of these tougher clinical scenarios. However, a better approach to help improve the cardiovascular workup of this minute patient population is to pursue an interdisciplinary approach, which is a collaboration between anesthesiology, cardiology, and surgery on whether further cardiac workup is needed and if it will actually change the clinical course of the patient.

There are many types of cardiac stress tests: exercise stress testing, which includes the exercise ECG stress test, exercise echocardiography stress test, exercise myocardial perfusion imaging test (nuclear), cardiopulmonary exercise test; and pharmacological stress testing which includes dobutamine echocardiography stress test, vasodilator (dipyridamole, adenosine, regadenoson) myocardial perfusion imaging (nuclear) stress test [12]. While there are published recommendations and appropriate use criteria for specific types of cardiac stress tests [13-16], there is no consensus or recommendation about which type of cardiac stress test to choose preoperatively. Only the most recent Stepwise Approach to Perioperative Cardiac Assessment Treatment algorithm from the 2014 ACC/AHA Guidelines on Perioperative Cardiovascular Evaluation and Management of Patients Undergoing Noncardiac Surgery recommends specifically a pharmacologic stress test [2]. The previous 2002 and 2007 iterations did not specify a type of cardiac stress test, and therefore the choice of type of cardiac stress test was up to the ordering clinician but frequently dictated by whether the patient has physical or orthopedic comorbidities that limited them from exercising. However, there are a few subsets of patients where a specific modality of cardiac stress test is preferred according to the ACC/AHA guidelines [2]. Exercise ECG stress testing is not the optimal choice for patients with resting ECG abnormalities as they often make the test invalid. In addition, due to the septal wall motion of a LBBB or RV pacing, the optimal test is a pharmacological vasodilator myocardial perfusion stress test.

Among the exercise ECG stress tests ordered in this study, $42 \%$ had resting abnormalities that invalidated them and should have warranted a different cardiac stress testing modality. In patients who had a resting ECG with LBBB or RV pacing, only $41 \%$ of the cardiac stress tests were pharmacologic vasodilator myocardial perfusion stress tests. This suggests that perioperative physicians ordering cardiac stress tests may need education on the optimal cardiac stress test modality. Our institution has already been implementing education of all anesthesiologists rotating in the preoperative clinic with the help of an establishment of a core staff of anesthesiologists with expertise in preoperative evaluation and management. We believe this is demonstrated by the fact no inappropriately ordered exercise ECG stress tests were ordered after 2011. The education efforts at our institution by the core staff of preoperative anesthesiologists has been by creating dedicated grand rounds yearly on preoperative medicine and through frequent staff communications on updates and changes to guidelines for preoperative workup. In addition, quality assessment and quality improvement through projects like this retrospective review provide areas of where education should be focused. Our Anesthesiology Department has a residency program, and daily dedicated teaching on topics of preoperative medicine 
and workup is provided to all anesthesiology residents when they rotate through the preoperative center. Also, our institution has created a perioperative medicine fellowship in anesthesiology to allow individuals who want additional training in the field gain extra experience.

This study focused on the indications for ordering cardiac stress tests rather than on the results of the cardiac stress tests and their impact on clinical management. As previously mentioned, those later inquiries are very important but far outside the scope and capabilities of this study. Morgan et al examined [17] the utility of dobutamine stress echocardiograms during the preoperative evaluation of patients scheduled for non-cardiac surgery. That study reviewed 85 patients and focused on the utility of the positive cardiac stress tests to guide further intervention. While the study was useful, to properly assess the utility of preoperative cardiac stress tests on further clinical management, an extremely large multi-center study would be required to obtain a sufficient number of positive cardiac stress tests resulting in subsequent interventions. For this reason, examination of the results of the cardiac stress tests and their effect on clinical management was not examined.

A limitation to our study is that it was retrospective from a single center. While the BWH PEC is a high-volume clinic that was seeing over 25,000 patients a year during the study period and has been in place for a relatively long time, this study examines only the cardiac stress tests ordering patterns of the anesthesiologists from single institution. This cannot be generalized to all anesthesiologists at all centers.

\section{Conclusions}

This study shows that most preoperative cardiac stress tests are ordered by anesthesiologists according to societal or institutional guidelines. The type of preoperative cardiac stress test ordered by anesthesiologists is not always optimal. Education regarding choice of cardiac stress modality has improved over time but continues to benefit from ongoing departmental continuing educational initiatives. In addition, a significant portion of cardiac stress tests are ordered based on clinical judgment likely due to the lack of guidelines and recommendations on many commonly encountered preoperative patient scenarios.

\section{Grant Support}

No grant support or other assistance.

\section{Conflict of Interest}

None.

\section{Disclosures}

The submitted manuscript represents original work that has not been published or is not being considered for publication else- where. All authors have contributed, participated sufficiently in the intellectual content and analysis of the data. Each author has reviewed the final version of the manuscript and approved it for publication. The datasets used and analyzed during the current study are not publicly available due to their current use on other research projects but are available from the corresponding author on reasonable request. The authors declare that there was no funding for this research.

\section{References}

1. Blitz JD, Kendale SM, Jain SK, Cuff GE, Kim JT, Rosenberg AD. Preoperative evaluation clinic visit is associated with decreased risk of in-hospital postoperative mortality. Anesthesiology. 2016;125(2):280-294.

2. Fleisher LA, Fleischmann KE, Auerbach AD, Barnason SA, Beckman JA, Bozkurt B, Davila-Roman VG, et al. 2014 ACC/AHA guideline on perioperative cardiovascular evaluation and management of patients undergoing noncardiac surgery: a report of the American College of Cardiology/American Heart Association Task Force on Practice Guidelines. Circulation. 2014;130(24):e278333.

3. Fleisher LA, Beckman JA, Brown KA, Calkins H, Chaikof EL, Fleischmann KE, Freeman WK, et al. ACC/ AHA 2007 guidelines on perioperative cardiovascular evaluation and care for noncardiac surgery: a report of the American College of Cardiology/American Heart Association Task Force on Practice Guidelines (Writing Committee to Revise the 2002 Guidelines on Perioperative Cardiovascular Evaluation for Noncardiac Surgery) developed in collaboration with the American Society of Echocardiography, American Society of Nuclear Cardiology, Heart Rhythm Society, Society of Cardiovascular Anesthesiologists, Society for Cardiovascular Angiography and Interventions, Society for Vascular Medicine and Biology, and Society for Vascular Surgery. J Am Coll Cardiol. 2007;50(17):e159-241.

4. Eagle KA, Berger PB, Calkins H, Chaitman BR, Ewy GA, Fleischmann KE, Fleisher LA, et al. ACC/AHA guideline update for perioperative cardiovascular evaluation for noncardiac surgery - executive summary: a report of the American College of Cardiology/American Heart Association Task Force on Practice Guidelines (Committee to Update the 1996 Guidelines on Perioperative Cardiovascular Evaluation for Noncardiac Surgery). J Am Coll Cardiol. 2002;39(3):542-553.

5. Morris F, Brady WJ. ABC of clinical electrocardiography: Acute myocardial infarction-Part I. BMJ. 2002;324(7341):831-834.

6. Edhouse J, Brady WJ, Morris F. ABC of clinical electrocardiography: Acute myocardial infarction-Part II. BMJ. 2002;324(7343):963-966.

7. Correll DJ, Hepner DL, Chang C, Tsen L, Hevelone ND, Bader AM. Preoperative electrocardiograms: patient factors predictive of abnormalities. Anesthesiology. 2009;110(6):1217-1222.

8. Lee TH, Marcantonio ER, Mangione CM, Thomas EJ, 
Polanczyk CA, Cook EF, Sugarbaker DJ, et al. Derivation and prospective validation of a simple index for prediction of cardiac risk of major noncardiac surgery. Circulation. 1999;100(10):1043-1049.

9. Fihn SD, Gardin JM, Abrams J, Berra K, Blankenship JC, Dallas AP, Douglas PS, et al. 2012 ACCF/AHA/ ACP/AATS/PCNA/SCAI/STS Guideline for the diagnosis and management of patients with stable ischemic heart disease: a report of the American College of Cardiology Foundation/American Heart Association Task Force on Practice Guidelines, and the American College of Physicians, American Association for Thoracic Surgery, Preventive Cardiovascular Nurses Association, Society for Cardiovascular Angiography and Interventions, and Society of Thoracic Surgeons. J Am Coll Cardiol. 2012;60(24):e44-e164.

10. Wilhelmsen L, Rosengren A, Hagman M, Lappas G. "Nonspecific" chest pain associated with high long-term mortality: results from the primary prevention study in Goteborg, Sweden. Clin Cardiol. 1998;21(7):477-482.

11. Jousilahti P, Vartiainen E, Tuomilehto J, Puska P. Sex, age, cardiovascular risk factors, and coronary heart disease: a prospective follow-up study of 14786 middle-aged men and women in Finland. Circulation. 1999;99(9):11651172.

12. Arbab-Zadeh A. Stress testing and non-invasive coronary angiography in patients with suspected coronary artery disease: time for a new paradigm. Heart Int. 2012;7(1):e2.

13. Gibbons RJ, Balady GJ, Bricker JT, Chaitman BR, Fletcher GF, Froelicher VF, Mark DB, et al. ACC/AHA 2002 guideline update for exercise testing: summary article. A report of the American College of Cardiology/American Heart Association Task Force on Practice Guidelines (Committee to Update the 1997 Exercise Testing Guidelines). J Am Coll Cardiol. 2002;40(8):1531-1540.

14. Hendel RC, Berman DS, Di Carli MF, Heidenreich PA, Henkin RE, Pellikka PA, Pohost GM, et al. ACCF/ASNC/
ACR/AHA/ASE/SCCT/SCMR/SNM 2009 Appropriate Use Criteria for Cardiac Radionuclide Imaging: A Report of the American College of Cardiology Foundation Appropriate Use Criteria Task Force, the American Society of Nuclear Cardiology, the American College of Radiology, the American Heart Association, the American Society of Echocardiography, the Society of Cardiovascular Computed Tomography, the Society for Cardiovascular Magnetic Resonance, and the Society of Nuclear Medicine. J Am Coll Cardiol. 2009;53(23):2201-2229.

15. American College of Cardiology Foundation Appropriate Use Criteria Task F, American Society of E, American Heart A, American Society of Nuclear C, Heart Failure Society of A, Heart Rhythm S, Society for Cardiovascular A, et al. ACCF/ASE/AHA/ASNC/HFSA/HRS/SCAI/ SCCM/SCCT/SCMR 2011 Appropriate Use Criteria for Echocardiography. A Report of the American College of Cardiology Foundation Appropriate Use Criteria Task Force, American Society of Echocardiography, American Heart Association, American Society of Nuclear Cardiology, Heart Failure Society of America, Heart Rhythm Society, Society for Cardiovascular Angiography and Interventions, Society of Critical Care Medicine, Society of Cardiovascular Computed Tomography, and Society for Cardiovascular Magnetic Resonance Endorsed by the American College of Chest Physicians. J Am Coll Cardiol. 2011;57(9):1126-1166.

16. Pellikka PA, Nagueh SF, Elhendy AA, Kuehl CA, Sawada SG, American Society of E. American Society of Echocardiography recommendations for performance, interpretation, and application of stress echocardiography. J Am Soc Echocardiogr. 2007;20(9):1021-1041.

17. Morgan PB, Panomitros GE, Nelson AC, Smith DF, Solanki DR, Zornow MH. Low utility of dobutamine stress echocardiograms in the preoperative evaluation of patients scheduled for noncardiac surgery. Anesth Analg. 2002;95(3):512-516, table of contents. 